\title{
Role of correlations in two-neutron transfer reactions
}

\author{
Jesus Lubian ${ }^{1, *}$, Jonas L. Ferreira, Roberto Linares, Erica N. Cardozo, Barbara Paes, Francesco Cappuzzello ${ }^{2}$, \\ Diana Carbone ${ }^{2}$, Manuela Cavallaro ${ }^{2}$, Uiran U. da Silva ${ }^{3}$, Ruben Lichtenthäler ${ }^{3}$ \\ ${ }^{1}$ Instituto de Física, Universidade Federal Fluminense, Niterói, Rio de Janeiro 24210-340, Brazil²INFN, Laboratori Nazionali del Sud, I- \\ 95125, Catania, Italy \\ ${ }^{3}$ Departamento de Física Nuclear, Universidade de São Paulo, C.P. 66318, 05389-970, São Paulo, Brazil
}

\begin{abstract}
This work presents theoretical results compared with the experimental data for the two-neutron transfer angular distribution in which a beam of ${ }^{18} \mathrm{O}$ nucleus, at $84 \mathrm{MeV}$ incident energy, has collided on the ${ }^{13} \mathrm{C},{ }^{28} \mathrm{Si}$, and ${ }^{64} \mathrm{Ni}$ targets. The two-neutron transfer in the ${ }^{9} \mathrm{Be}\left({ }^{7} \mathrm{Be},{ }^{9} \mathrm{Be}\right){ }^{7} \mathrm{Be}$ reaction, at $23.1 \mathrm{MeV}$ incident energy, was also analyzed. The main goal was to verify the relevance of the pairing correlation of the two transferred neutrons on the cross sections and to show its role when both neutrons are transferred to states with a low and high degree of collectivity.
\end{abstract}

\section{Introduction}

The study of pairing correlation in two-nucleon transfer reaction has intensively been performed in the last decades. In the past, this kind of direct reactions was usually probed by the $(t, p)$ and $(p, t)$ reactions. Due to restrictive radioprotection rules, these reactions were prohibited. Another alternative reaction was proposed in the Istituto Nazionale di Fisica Nucleare - Laboratori Nazionali del Sud (Italy) by considering the $\left({ }^{18} \mathrm{O},{ }^{16} \mathrm{O}\right)$ reaction, once the ${ }^{18} \mathrm{O}$ nucleus can be considered as composed by an inert core of ${ }^{16} \mathrm{O}$ and two valence neutrons. In this sense, some reactions were measured considering the ${ }^{18} \mathrm{O}$ nucleus as projectile at $84 \mathrm{MeV}$ incident energy, and ${ }^{12,13} \mathrm{C}[1,2],{ }^{16} \mathrm{O}[3,4],{ }^{28} \mathrm{Si}[5]$ and ${ }^{64} \mathrm{Ni}[6]$ as target to analyze the feasibility of the $\left({ }^{18} \mathrm{O},{ }^{16} \mathrm{O}\right)$ reaction as substitute of the $(t, p)$ one, and then to investigate the role of the pairing correlation of both transferred neutrons in the transfer reaction cross sections obtained performing coupled reaction channel calculations [7]. The ${ }^{12} \mathrm{C}\left({ }^{18} \mathrm{O},{ }^{16} \mathrm{O}\right){ }^{14} \mathrm{C}$ reaction has experimentally shown, similarly to $(t, p)$, the feasibility in populating the twoneutron configuration of the ${ }^{14} \mathrm{C}$ nucleus.

In particular, the two-neutron transfer in the ${ }^{18} \mathrm{O}+{ }^{16} \mathrm{O}$ reaction includes the complexity of dealing with identical ingoing and outgoing partitions, as well as the recently studied two-neutron transfer in the ${ }^{9} \mathrm{Be}+{ }^{7} \mathrm{Be}$ reaction [8] at $23 \mathrm{MeV}$, and the alpha-particle transfer in the ${ }^{16} \mathrm{O}+{ }^{12} \mathrm{C}$ at 80 and $84 \mathrm{MeV}$ incident energy [9]. The complexity appears because the elastic scattering and elastic transfer are indistinguishable processes, and they can compete. In these cases, the quantum interference effect between elastic scattering and elastic transfer should be explicitly taken into account in the calculations.

Another important aspect of the transfer reactions is their high selectivity, which is very important to study the nuclear structure of the involved nuclei. By means of these reactions, it is possible to obtain spectroscopic information as the spectroscopic amplitudes that are connected to the strength of each single- or two-particle component of the nuclear wave functions (or even to the alpha-particle component). As it is well known, the two valence particles may be simultaneously or sequentially transferred from the projectile to the target in stripping reactions, and these processes may compete with each other. While the simultaneous two-nucleon transfer reactions (or one-step) reveal the two-particle component of the nuclear wave functions, the sequential twonucleon transfer (or two-step) raises their one-particle nature. In this work, we discuss the competition between the collectivity and the pairing correlation in the states populated by the two transferred particles. The twoneutron transfer angular distribution has been quite well described by the theoretical calculation for some specific measured channels. The theoretical results show that the states with high collectivity are preferably accessed by the sequential two-neutron transfer process suggesting that the correlation of the two transferred neutrons was broken by the collectivity of the populated final state.

This work is organized as ${ }^{18} \mathrm{O}$ follows. The next section is devoted to the results and discussions while in the last section, we give some remarks and show some perspectives on our work guidelines.

\section{Results}

The more straightforward theoretical treatment involving the rearrangement reaction can be reached by considering only the ground state of the partner nuclei in the initial and final partition, as well as, assuming the coupling between the partitions as weak. In this context, the distorted wave Born approximation (DWBA) approach might be applied, once the coupling interaction is regarded in the first order. On the other hand, if the coupling interaction between the partitions is extended to higher-orders, the coupled reaction channel (CRC) method will be invoked. Moreover, in two-neutron stripping transfer reaction like, for instance, $a(b+2 n)+$ $A \rightarrow b+B(A+2 n)$ the transfer amplitude can be determined by the equation

$$
T_{\alpha \rightarrow \gamma}=\left\langle\chi_{\gamma}^{(-)}\left|W_{\alpha \gamma}\right| \chi_{\alpha}^{(+)}\right\rangle
$$

\footnotetext{
Corresponding author: lubian@if.uff.br
} 
so that the residual interaction responsible to the transfer process is written as $W_{\alpha}=U(R)+v\left(\boldsymbol{r}_{1}\right)+v\left(\boldsymbol{r}_{2}\right)-$ $U\left(R_{\alpha}\right)$. In addition, the matrix elements defined by $W_{\alpha \gamma} \equiv\left\langle\phi_{\gamma}\left|W_{\alpha}\right| \phi_{\alpha}\right\rangle$ contains all the structure information of the collision partners. $\chi_{\alpha}{ }^{(+)}$and $\chi_{\gamma}{ }^{*(-)}$ represent the wave functions of the relative motion in the entrance and final partitions, respectively, with the $\alpha$ and $\gamma$ being all the quantum numbers needed to specify the scattering state. In the collision, the target interacts with the core (in which the two valence neutrons are bound) by mean of the $U(R)$ interaction, and with each valence particles by $v\left(\boldsymbol{r}_{1}\right)$ and $v\left(\boldsymbol{r}_{2}\right) . U\left(R_{\alpha}\right)$ represents the interaction between projectile and target nuclei concerning the center of mass coordinate $R_{\alpha}$. Moreover, while $U(R)$ and $U\left(R_{\alpha}\right)$ are complex potentials, the $v\left(\boldsymbol{r}_{1}\right)$ and $v\left(\boldsymbol{r}_{2}\right)$ interactions are assumed to be real. From the transfer amplitudes we can determine the transfer angular distribution given by

$$
\frac{d \sigma_{\alpha \rightarrow \gamma}}{d \Omega}=\frac{\mu_{\alpha} \mu_{\gamma}}{\left(2 \pi h^{2}\right)^{2}} \frac{k_{\gamma}}{k_{\alpha}} \frac{1}{\left(2 I_{a}+1\right)\left(2 I_{A}+1\right)}\left|T_{\alpha \rightarrow \gamma}\right|^{2},
$$

where $\mu_{i}$ and $k_{i}$ are the reduced masses and the wave number in partition $i$. In the two-particle transfer, the T amplitude given in equation (1) is explicitly represented by two terms. The first, corresponds to the direct twoparticle transfer, in which the two particles are transferred from the initial partition to the final one, while, the second term, is related to the sequential twoparticle transfer, where both valence particles are transferred one by one passing through an intermediate partition, let say $\beta$. In this case, the equation (1) is represented as $T_{\alpha \rightarrow \gamma}=T_{\alpha \rightarrow \gamma}^{(1)}+T_{\alpha \rightarrow \beta \rightarrow \gamma}^{(2)}$ [7]. The direct and sequential two-particle transfer can compete with each other and both terms should be considered in the same calculation. However, it is essential to consider each transfer mechanism to study the degree of correlation between the transferred particles.

In the present work, the two-neutron transfer angular distributions were calculated considering the finite-range coupled reaction channel method using the Fresco code [10].

To perform microscopic transfer reaction calculations, it is important to know the one and two particle spectroscopic amplitudes, as well as, to use a parameter-free optical potential (like a double folding potential). In this work, the theoretical transfer calculations were performed using the São Paulo double folding potential [11] in the real and imaginary parts of the optical potential $\left(\mathrm{U}(\mathrm{R})=\left(\mathrm{N}+i \mathrm{~N}_{i}\right) \mathrm{V}^{\mathrm{SP}}(\mathrm{R})\right)$. In the ingoing partition, the imaginary part was normalized by a factor 0.6 [12], which consider that the more relevant couplings to the bound states were explicitly included, but some dissipation to continuum occurs. On the other hand, in the outgoing partition, the imaginary part was multiplied by a factor 0.78 [13], once no couplings were considered. The spectroscopic amplitudes concerning the one- and two-neutron wave function overlaps were derived from the shell model calculation by using the NuShellX code [14]. The single-particle wave functions were generated considering a Woods-Saxon potential whose depth was varied to fit the experimental biding energies of one and two neutrons.

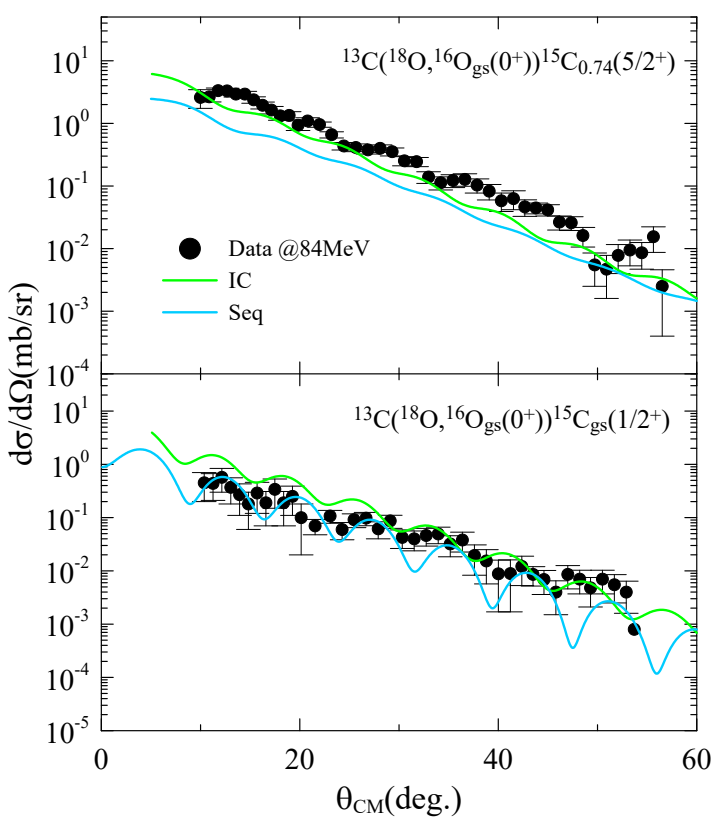

Fig. 1. Comparison of the experimental data and theoretical results corresponding to the ${ }^{13} \mathrm{C}\left({ }^{18} \mathrm{O},{ }^{16} \mathrm{O}\right){ }^{15} \mathrm{C}$. IC means independent coordinates CRC calculations. Seq stands for the sequential CCBA calculations.

To probe the pairing correlations in the ${ }^{12,13} \mathrm{C}\left({ }^{18} \mathrm{O},{ }^{16} \mathrm{O}\right){ }^{14,15} \mathrm{C},{ }^{16} \mathrm{O}\left({ }^{18} \mathrm{O},{ }^{16} \mathrm{O}\right){ }^{18} \mathrm{O},{ }^{28} \mathrm{Si}\left({ }^{18} \mathrm{O},{ }^{16} \mathrm{O}\right){ }^{30} \mathrm{Si}$, and ${ }^{64} \mathrm{Ni}\left({ }^{18} \mathrm{O},{ }^{16} \mathrm{O}\right){ }^{66} \mathrm{Ni}$ reactions, we have separately analyzed the direct and sequential two-neutron transfer reactions. In particular, the microscopic independent coordinates scheme was considered to analyze the direct two-neutron transfer in finite range coupled reaction channel calculations. Here, both valence particles are treated as particles spatially separated and simultaneously transferred from the initial to the final state. In this case, the spectroscopic amplitudes correspond to two-particle configurations. Details about this method can be found in Ref. [10]. On the other hand, we also considered the sequential process in which the two neutrons are transferred one by one passing through an intermediate partition. In these transfer calculations, high-order couplings between states inside the partition and to first-order between different partitions were considered. This means that the coupled channel Born approximation was employed for the sequential twoneutron transfer calculations. In a full quantum treatment, the direct and sequential two-neutron transfer should be considered together in the same calculation.

In the two-neutron transfer reaction studies mentioned above, the cross section for the direct and sequential processes that populated the ground state of the residuals ${ }^{14,15} \mathrm{C},{ }^{18} \mathrm{O},{ }^{30} \mathrm{Si}$, and ${ }^{66} \mathrm{Ni}$ nuclei had the same order of magnitude. The result of this study is shown in Figures 1,2, and 3, in which the ${ }^{18} \mathrm{O}$ was the projectile impinging on light- and medium-mass targets. Conversely, for the two-neutron transfer in the ${ }^{206} \mathrm{~Pb}\left({ }^{18} \mathrm{O},{ }^{16} \mathrm{O}\right){ }^{208} \mathrm{~Pb}$ reaction [16] the cross section of the sequential process was larger than the direct one. 


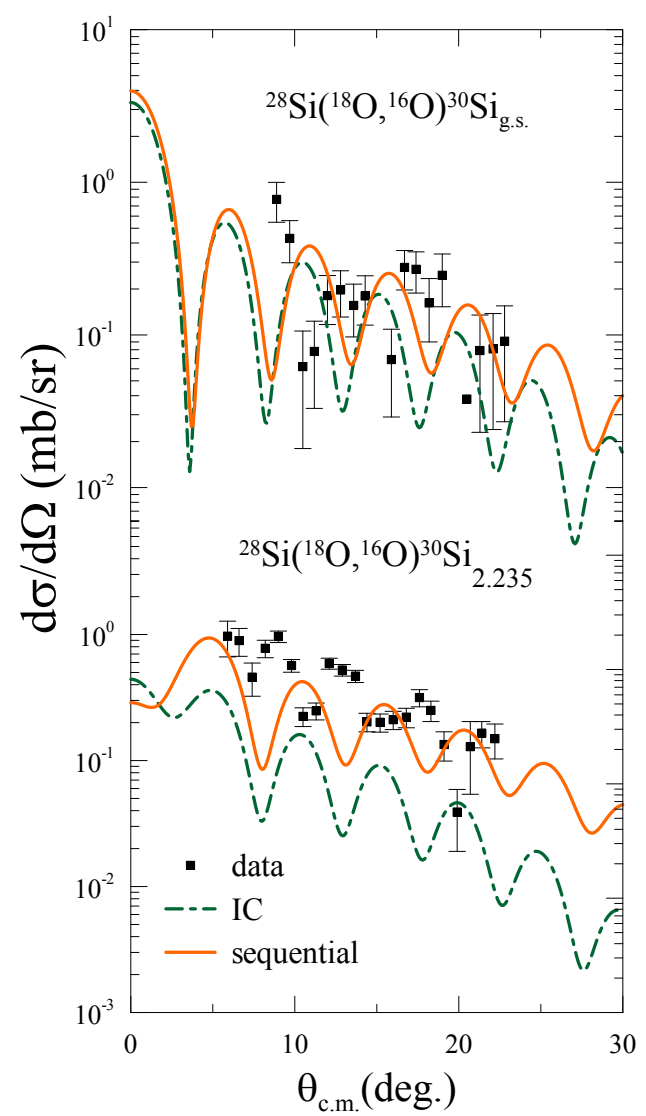

Fig. 2. Comparison of the experimental data and theoretical results corresponding to the ${ }^{28} \mathrm{Si}\left({ }^{18} \mathrm{O},{ }^{16} \mathrm{O}\right){ }^{30} \mathrm{Si}$. IC means independent coordinates CRC calculations. Sequential stands for the sequential CCBA calculations.

The scenario is a little bit different when the two neutrons populate some excited states of the residual nuclei with strong collectivity. In this case, the correlation amount a considerable number of nucleons is more relevant, and the pairing correlation does not appear to be very important. In this situation, the sequential process becomes the most suitable reaction mechanism. On the other hand, the excited states with lower collectivity were preferably populated by the direct two-neutron transfer in the light residual nuclei ${ }^{14,15} \mathrm{C}$ and ${ }^{18} \mathrm{O}$. In Figure 1, we just show the results for the angular distribution populating the ground $\left(1 / 2^{+}\right)$and first excited $\left(5 / 2^{+}\right)$states of the ${ }^{15} \mathrm{C}$ nucleus. For the light-medium ${ }^{30} \mathrm{Si}$ and medium ${ }^{66} \mathrm{Ni}$ mass residual nuclei, the sequential two-neutron transfer was more likely to occur, as can be seen in Figures 2 and 3. This behavior has been associated with the competition between the collective and pairing residual interactions. In the ${ }^{13} \mathrm{C}\left({ }^{18} \mathrm{O},{ }^{16} \mathrm{O}\right){ }^{15} \mathrm{C}$ some evidence of the existence of giant pairing vibration [15] were found which are produced by the coherent particle-particle excitation during the removal or addition of two nucleons with relative motion characterized by the s-wave.
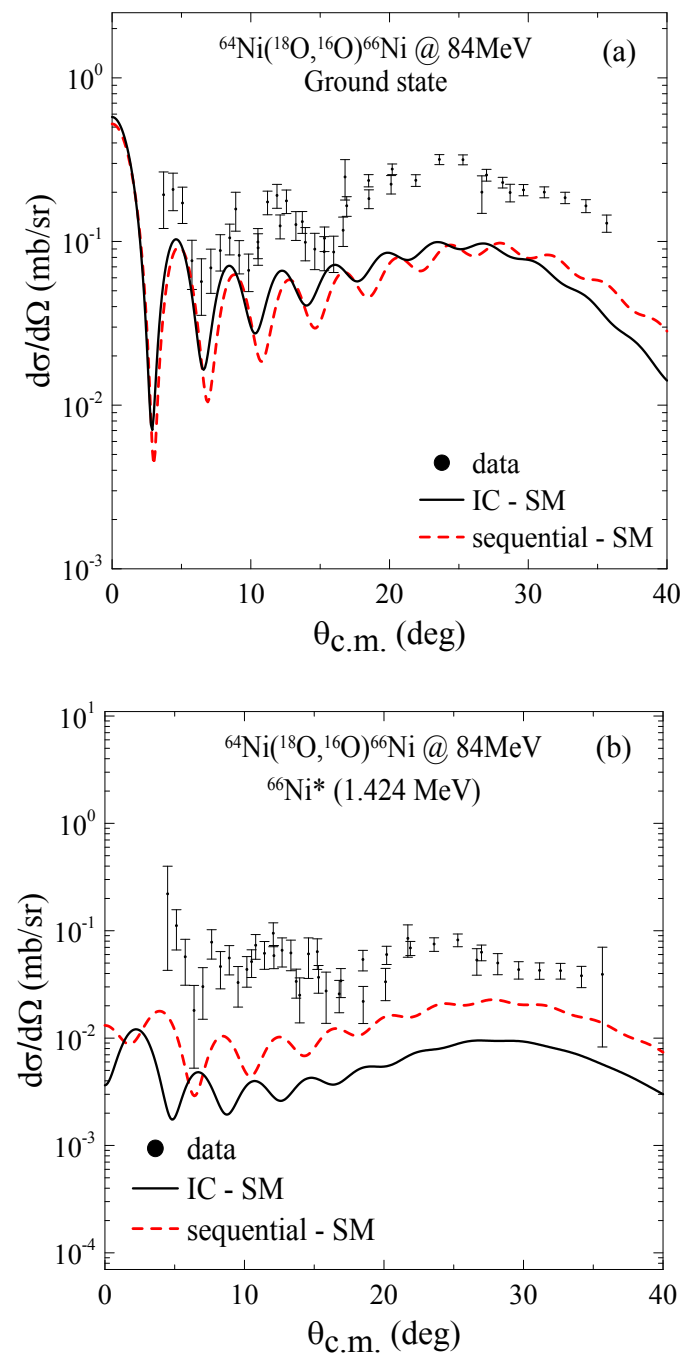

Fig. 3. Comparison of the experimental data and theoretical results corresponding to the ${ }^{64} \mathrm{Ni}\left({ }^{18} \mathrm{O},{ }^{16} \mathrm{O}\right){ }^{66} \mathrm{Ni}$. IC means independent coordinates CRC calculations. Sequential stands for the sequential CCBA calculations. SM means that the spectroscopic amplitudes are derived from shell-model calculations.

The ${ }^{9} \mathrm{Be}\left({ }^{7} \mathrm{Be},{ }^{9} \mathrm{Be}\right){ }^{7} \mathrm{Be}[8]$ elastic transfer reaction shows features similar to the $\left({ }^{18} \mathrm{O},{ }^{16} \mathrm{O}\right)[3,4]$ elastic transfer mentioned above, in the sense that the direct two-neutron mechanism is very important (in fact, in this case, it is the preferred mechanism). In this reaction, the elastic scattering and elastic transfer are indistinguishable processes, once the ingoing and outgoing partitions are the same. Therefore, the quantum effect of interference between both processes have been taken into account. It was shown that, while the elastic scattering is the predominant process at forward angles, the elastic-transfer is more likely to occur at backward angles. On the other hand, in the alpha-transfer reaction ${ }^{12} \mathrm{C}\left({ }^{16} \mathrm{O},{ }^{12} \mathrm{C}\right){ }^{16} \mathrm{O}$, it is observed a strong competition between the elastic scattering and elastic transfer processes at backward angles. In Figure 4, we show the comparison between experimental data and theoretical predictions for the angular distribution concerning the quasi-elastic collision ${ }^{7} \mathrm{Be}+{ }^{9} \mathrm{Be}$. One can observe that the direct two-neutron transfer is the most important process at backward angles when we compared with the sequential one. 


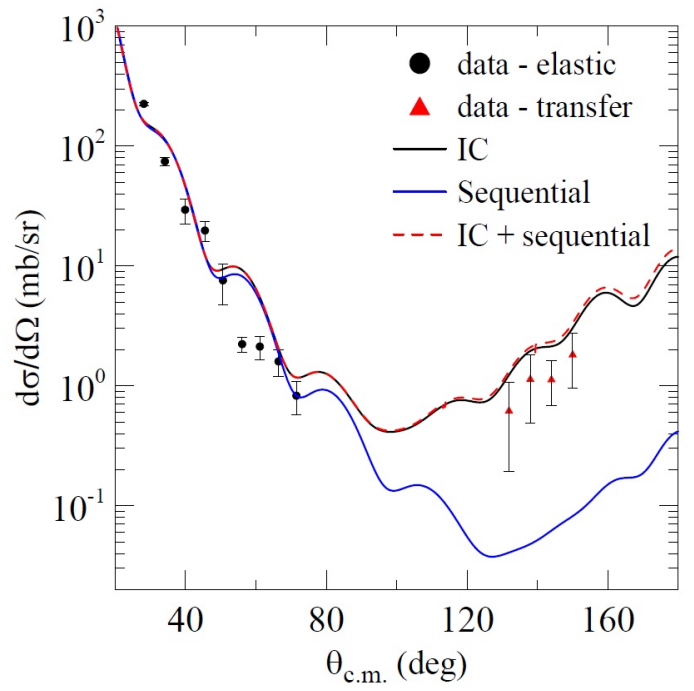

Fig. 4. Comparison of the experimental data and theoretical predictions corresponding to the elastic scattering and elastictransfer angular distributions in the ${ }^{7} \mathrm{Be}+{ }^{9} \mathrm{Be}$ collision at 23.1 $\mathrm{MeV}$.

\section{Conclusions}

In this work, we present some measured angular distributions corresponding to the two-neutron transfer reaction in comparison with the theoretical calculation by using the finite range coupled reaction channel method. The data for the ${ }^{12,13} \mathrm{C}\left({ }^{18} \mathrm{O},{ }^{16} \mathrm{O}\right){ }^{14,15} \mathrm{C}$, ${ }^{16} \mathrm{O}\left({ }^{18} \mathrm{O},{ }^{16} \mathrm{O}\right){ }^{18} \mathrm{O},{ }^{28} \mathrm{Si}\left({ }^{18} \mathrm{O},{ }^{16} \mathrm{O}\right){ }^{30} \mathrm{Si}$ and ${ }^{64} \mathrm{Ni}\left({ }^{18} \mathrm{O},{ }^{16} \mathrm{O}\right){ }^{66} \mathrm{Ni}$ reactions, at $84 \mathrm{MeV}$ incident energy, were measured at Istituto Nazionale di Fisica Nucleare - Laboratori Nazionali del Sud (Italy). Besides, the experimental data for the ${ }^{9} \mathrm{Be}\left({ }^{7} \mathrm{Be},{ }^{9} \mathrm{Be}\right){ }^{7} \mathrm{Be}$ reaction were showed and compared with the theoretical results. This data have been measured at São Paulo University using the RIBRAS (Radioactive Ion Beams in Brazil) facility. The results show the importance of the pairing correlation between the two valence neutrons in the transfer reactions.

In final states with high collectivity, the sequential two-neutron transfer is favored. On the other hand, nuclear states with low collectivity have been populated through the direct two-neutron transfer.

The Brazilian authors acknowledge the partial financial support from CNPq, FAPERJ, and CAPES and fromINCT-FNA (Instituto Nacional de Ciência e Tecnologia- Física Nuclear e Aplicações).

\section{References}

1. M. Cavallaro, F. Cappuzzello, M. Bondi, D. Carbone, V.N. Garcia, A. Gargano, S. M. Lenzi, J. Lubian, C. Agodi,F.Azaiez, et al., Phys. Rev. C 88, 054601, (2013).

2. D. Carbone, J.L. Ferreira, F. Cappuzzello, J. Lubian, C. Agodi, M. Cavallaro, A. Foti, A. Gargano, S.M. Lenzi, R. Linares, G. Santagati, Phys. Rev. C 95, 034603, (2017).

3. M.J. Ermamatov, F. Cappuzzello, J. Lubian, M. Cubero, C. Agodi, D. Carbone, M. Cavallaro, J.L.
Ferreira, A. Foti, V.N. Garcia, A. Gargano, J. A. Lay, S.M. Lenzi, R. Linares, G. Santagati, and A. Vitturi, Phys. Rev. C 94, 024610, (2016).

4. M.J. Ermamatov, R. Linares, J. Lubian, J.L. Ferreira, F. Cappuzzello, D. Carbone, M. Cavallaro, P.N. de Faria, A. Foti, G. Santagati, and V.A.B. Zagatto, Phys. Rev. C 96, 044603, (2017).

5. E.N. Cardozo, J. Lubian, R. Linares, F. Cappuzzello, D. Carbone, M. Cavallaro, J. L. Ferreira, A. Gargano, B.Paes, and G. Santagati, Phys. Rev. C 97, 06461, (2018).

6. B. Paes, G. Santagati, R.M. Vsevolodovna, F. Cappuzzello, D. Carbone, E.N. Cardozo, M. Cavallaro, H.Garcia-Tecocoatzi, A. Gargano, J.L. Ferreira, S.M.Lenzi, R. Linares, E. Santopinto, A. Vitturi, and J. Lubian, Phys. Rev. C 96, 044612, (2017).

7. G. R. Satchler, Direct Nuclear Reactions (Oxford University Press, Oxford, 1983).

8. U. Umbelino, K.C.C. Pires, R. Lichtenthaler, V. Scarduelli, G.A. Scotton, A. L'epine-Szily, V. Guimarães, J. Lubian, B. Paes, J.L. Ferreira, M.A. G. Alvarez, J.M.B. Shorto, S. Appannababu, M. Assunção, R.P. Condori, V. Morcelle, Phys. Rev. C 99, 064617, (2019).

9. J.L. Ferreira, J. Lubian, R. Linares, M.J. Ermamatov, H. Yépez-Martínez and P.O.Hess, Eur. Phys. J. A 55, 94, (2019).

10. I.J. Thompson, Comput. Phys. Rep. 7, 167, (1988).

11. L.C. Chamon, D. Pereira, M.S. Hussein, M.A. Cândido Ribeiro, and D. Galetti, Phys. Rev. Lett. 79, 5218, (1997).

12. D. Pereira, J. Lubian, J. Oliveira, D. Sousa, and L. Chamon, Phys. Lett. B 670, 330, (2009).

13. L.R. Gasques, L.C. Chamon, P.R.S. Gomes, and J. Lubian, Nucl. Phys. A 764, 135, (2006).

14. NuShellX for Windows and Linux http://www.garsington.eclipse.co.uk/.

15. F. Cappuzzello, D. Carbone, M. Cavallaro, M. Bondì,C.Agodi, F. Azaiez, A.Bonaccorso, A. Cunsolo, L. Fortunato, A. Foti, et al., Nat. Comm.6, 6743, (2015).

16. A. Parmar, Sonika, B.J. Roy, V. Jha, U.K. Pal, T. Sinha, S.K. Pandit, V.V. Parkar, K. Ramachandran, K. Mahata, S.Santra, A.K. Mohanty, Nucl. Phys. A 940, 167, (2015). 